\title{
Invasive mucormycosis during treatment for acute lymphoblastic leukaemia_-successful management of two life-threatening diseases
}

\author{
Andreas Trobisch ${ }^{1,2}$ (D) R. Marterer ${ }^{3} \cdot$ G. Gorkiewicz ${ }^{4} \cdot$ S. Flaschberger $^{5} \cdot$ H. Lackner $^{1} \cdot$ M. Seidel $^{1} \cdot$ D. Sperl ${ }^{1}$. \\ A. Karastaneva ${ }^{1} \cdot$ B. Kohlmaier ${ }^{1} \cdot$ M. Egger ${ }^{6} \cdot$ C. Urban $^{1} \cdot$ M. Benesch $^{1} \cdot$ V. Strenger ${ }^{1,6,7}$
}

Received: 16 March 2019 / Accepted: 11 June 2019 / Published online: 14 August 2019

(C) The Author(s) 2019

\begin{abstract}
A 5-year-old patient treated for acute lymphoblastic leukaemia (ALL) developed proven pulmonary invasive fungal disease (IFD) due to Actinomucor elegans. While completing ALL treatment according to AIEOP ALL protocol 2009 for further 15 months, antifungal treatment with liposomal amphotericin B and intermittent additional posaconazole was continued until immune reconstitution 7 months after the end of ALL treatment. Repeated imaging guided treatment decisions. Twenty-six and 19 months after the end of ALL treatment and antifungal treatment, respectively, the patient is still in the first complete remission and shows no signs of active invasive fungal disease (IFD).
\end{abstract}

Keywords ALL $\cdot$ Mucormycosis $\cdot$ IFD $\cdot$ Antimycotic treatment $\cdot$ Chemotherapy

$\begin{array}{ll}\text { Abbreviations } \\ \text { AIEOP } & \begin{array}{l}\text { Associazione Italiana Ematologia } \\ \text { Oncologia Pediatrica }\end{array} \\ \text { ALL } & \text { Acute lymphoblastic leukaemia } \\ \text { CNS } & \text { Central nervous system } \\ \text { CRP } & \text { C-reactive protein } \\ \text { CT } & \text { Computed tomography } \\ \text { ECIL } & \text { European Conference on Infections in Leukaemia }\end{array}$

V. Strenger

volker.strenger@medunigraz.at

1 Department of Paediatrics and Adolescent Medicine, Division of Paediatric Haematology/Oncology, Medical University, Graz, Austria

2 Department of Paediatrics and Adolescent Medicine, Division of Neonatology, Medical University, Graz, Austria

3 Department of Radiology, Division of Paediatric Radiology, Medical University Graz, Graz, Austria

4 Diagnostic \& Research Institute of Pathology, Medical University, Graz, Austria

5 Department of Paediatrics, Klinikum Klagenfurt am Wörthersee, Klagenfurt, Austria

6 Department of Paediatrics and Adolescent Medicine, Division of Paediatric Pulmonology and Allergology, Medical University, Graz, Austria

7 Research Unit Infectious Diseases in the Immunocompromised Host, Medical University, Graz, Austria
EORTC European Organisation for Research and Treatment of Cancer

FDG Fluoro-2-deoxyglucose

IFD Invasive fungal disease

IMI Invasive mould infection

MRI Magnetic resonance imaging

PCR Polymerase chain reaction

PET Positron emission tomography

\section{Introduction}

Invasive mould diseases remain an important cause of death in immunocompromised patients [1,2]. While invasive diseases with Aspergillus sp. have mortality rates of 20-50\%, mortality rates in mucormycosis range from 50 to $100 \%$ [3-5]. Differentiation between mucormycoses and other mould infections remains challenging, since these pathogens are difficult to culture and show similar radiological and clinical features leading from $4 \%$ to up to $90 \%$ of suspected mucormycosis cases not being confirmed until post-mortem examination $[1,4,5]$. Moreover, mucormycetes lack susceptibility to many antifungal agents and need higher dosages of amphotericin B [6, 7]. A mainstay of the successful management is the reversal of the underlying condition [4]. On the other hand, treatment of (haematologic) malignancies often leads to enhanced susceptibility for mucormycosis 
necessitating sophisticated management of these infections with concurrent further sufficient treatment of the malignant disease.

We describe the successful management of mucormycosis during ALL treatment.

\section{Case description}

A 5-year-old patient was treated for precursor B cell acute lymphoblastic leukaemia (ALL) according to AIEOP BFM ALL 2009 protocol for 7 months in the non-high-risk arm. For digestive decolonisation, he continuously received oral amphotericin B. Overall, he had received antileukaemic treatment for 25 weeks and 3 weeks of restarted steroid therapy in protocol IIA with dexamethasone $\left(10 \mathrm{mg} / \mathrm{m}^{2} /\right.$ day $)$, before he developed fever and CRP levels with a maximum of $200 \mathrm{mg} /$ L during neutropenia (neutrophilic leucocyte count $<500 /$ $\mu \mathrm{L}$ ); thus, ALL treatment was stopped. Chest X-ray performed 15 days after admission showed a diffuse spread infiltrate of up to $3 \mathrm{~cm}$ in the right middle to lower field and treatment with meropenem and clarithromycin was initiated and later switched to ceftazidim and linezolid. Furthermore, antimycotic treatment with caspofungin $(1 \mathrm{mg} / \mathrm{kg} / \mathrm{d})$ was initiated. Bronchoalveolar lavage (BAL) was sent for microbiological evaluation including auramine staining for

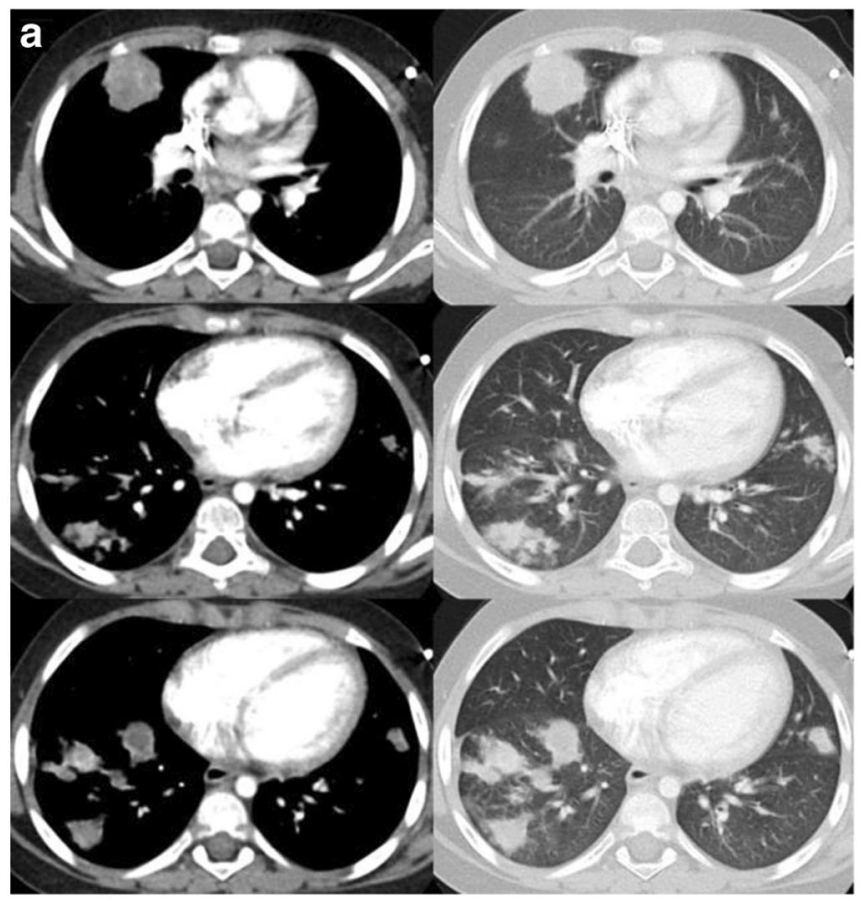

Fig. 1 Initial (a) CT scan of the chest and at last follow-up (b). Soft tissue windowing images are displayed in the left and corresponding lung windowing images in the right rows. In the initial series (a), multiple, partly enhancing nodules in both lungs with a maximum diameter of $2.1 \mathrm{~cm}$, predominantly in the lung periphery were detected. mycobacteria and calcofluor-white staining for fungus and revealed hyphae and weak growth of Aspergillus fumigatus. Neither fungal PCR from BAL fluid nor lung biopsy was performed at that time. Antifungal treatment was switched to liposomal amphotericin B (AmBisome ${ }^{\circledR}, 1 \mathrm{mg} / \mathrm{kg} /$ day) and voriconazole $(8 \mathrm{mg} / \mathrm{kg} /$ day $)$. Due to clinical and radiologic deterioration (Fig. 1), the patient was transferred to our University Hospital. Beta-D-glucan and galactomannan were both unremarkable, whereas CRP was still slightly elevated with $9.3 \mathrm{mg} / \mathrm{L}$. Uncomplicated thoracoscopic biopsy and subsequent histopathological examination revealed fungal hyphae, which were identified as Actinomucor elegans via fungal ITS PCR and sequencing (GenBank acc. no.: gb|FJ176396.1|; Identities: 312/313 bp (99\%)) representing proven invasive mucormycosis according to the EORTC criteria $[8,9]$. Therefore, voriconazole was switched to posaconazole suspension $(15 \mathrm{mg} / \mathrm{kg} /$ day $)$ and dosage of AmBisome ${ }^{\circledR}$ was increased to $10 \mathrm{mg} / \mathrm{kg} /$ day, with AmBisome ${ }^{\circledR}$-induced hypopotassaemia being substituted intravenously. Cranial magnet resonance imaging (MRI) did not show any signs of central nervous involvement of mucormycosis neither did the nasal sinuses show signs of obliteration. After 4 weeks without ALL treatment, an interval therapy with methotrexate $(0.5-0.7 \mathrm{mg} / \mathrm{kg}$ once weekly) and 6-mercaptopurine $(1.5 \mathrm{mg} / \mathrm{kg}$ daily) was administered for another month. Under continued antifungal treatment, we reinitiated therapy according to the AIEOP BFM ALL 2009

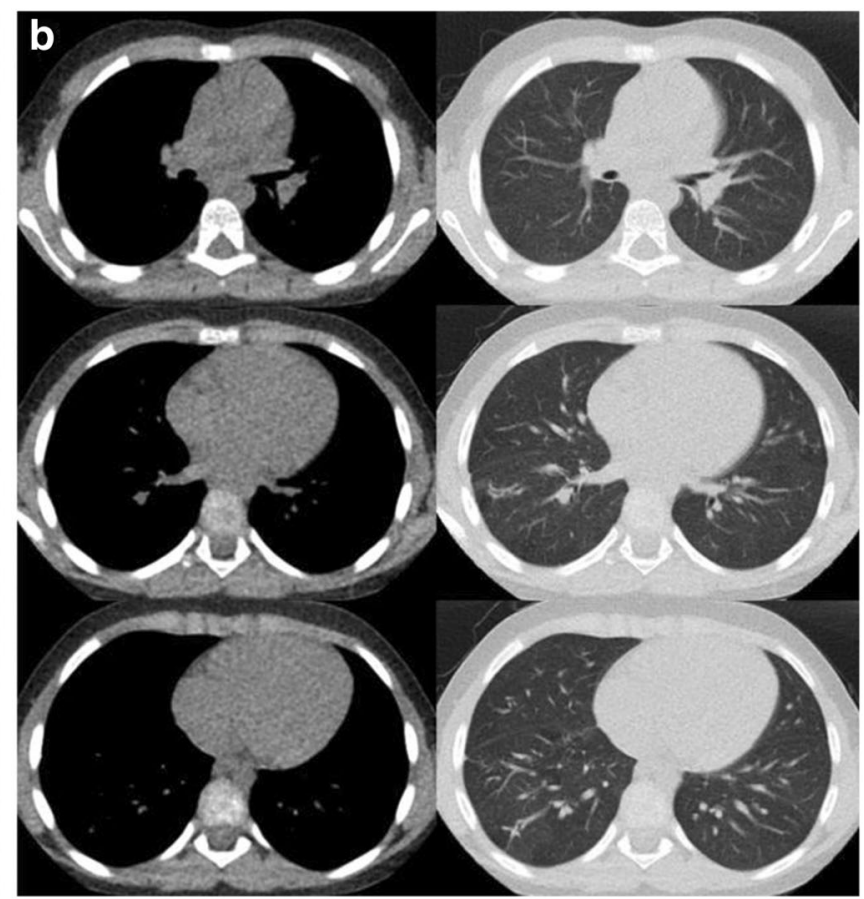

Furthermore, two consolidations with a maximum extent of $4.7 \mathrm{~cm}$ were present in the lingula and the right lower lobe. At last follow-up (b), only a few subtle residual changes in the lingula and the right lower lobe were seen 


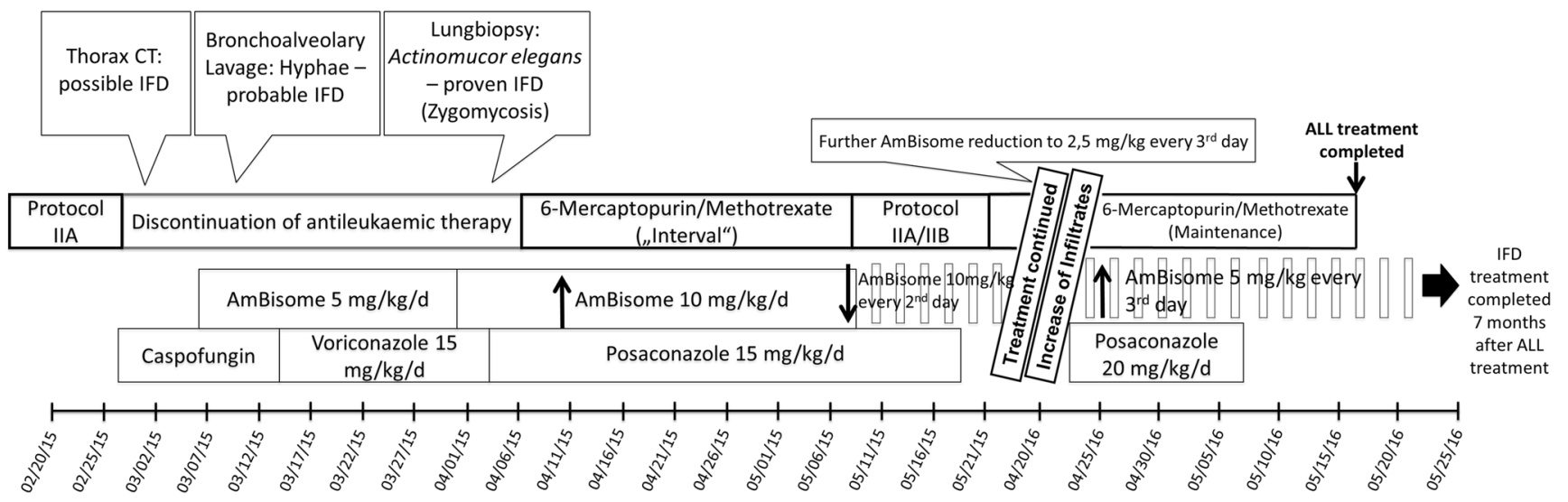

Fig. 2 Treatment timeline

protocol, completed block IIB and started maintenance therapy. Five weeks after the start of maintenance therapy, AmBisome ${ }^{\circledR}$ dosages were reduced to $10 \mathrm{mg} / \mathrm{kg} /$ day every 2 nd day. Another 8 weeks later, AmBisome ${ }^{\circledR}$ was reduced to $2.5 \mathrm{mg} / \mathrm{kg}$ /day every $3 \mathrm{rd}$ day due to practicability while posaconazole had to be discontinued due to lacking compliance (Fig. 2). The pulmonary mucormycosis was monitored every 2-6 months, either by thoracic CT or by MRI. Four months after the start of antifungal treatment, we additionally performed a PET-CT scan still showing multifocal metabolically active consolidations in both lungs. After 12 months of antifungal therapy with slight radiologic improvement, CT scan again showed an increase of infiltrates, leading to an increase in AmBisome ${ }^{\circledR}$ dosages to $5 \mathrm{mg} / \mathrm{kg} /$ day every $3 \mathrm{rd}$ day and reinitiation of posaconazole at an increased dosage of $20 \mathrm{mg} / \mathrm{kg} /$ day. The patient again refused posaconazole intake, so we had to stop combination therapy after only 2 weeks while continuing AmBisome ${ }^{\circledR}$ at $5 \mathrm{mg} / \mathrm{kg} /$ day every $3 \mathrm{rd}$ day. ALL treatment was terminated 22 months after diagnosis ( 2 months earlier than required) in order to improve the immune system. Flow cytometry further showed diminished B and T cell fractions after the termination of ALL treatment and CT/MRI as well as PET-CT scans still indicated infiltrates with reduced but still recognisable activity. Therefore, AmBisome ${ }^{\circledR}$ was given for another 7 months, until immunologic reconstitution (indicated by flow cytometry) and imaging showed significant improvement. Thirty months after the end of antileukaemic treatment and 23 months after the end of antifungal treatment, the patient is still in the first remission of ALL and recent thoracic CT and chest X-ray indicate further improvement with only signs of tissue scarring. An overview of our management is depicted in Fig. 2.

\section{Discussion}

We report the successful management of a 5-year-old patient treated for ALL, complicated by an opportunistic infection with Actinomucor elegans representing the first paediatric patient with this pathogen.

Actinomucor elegans belongs to the genus Actinomucor in the order Mucorales. So far, only 5 cases of infection with Actinomucor elegans in humans have been described in adults $[2,10,11]$. Two patients ( 1 immunocompetent, 1 recipient of an allogeneic stem cell transplantation for lymphoma) were described with sinusitis and were cured with debridement, irrigation and antifungal treatment [2, 12]. Two patients were described with soft tissue infections. One of them with underlying refractory aplastic anaemia died despite debridement and antifungal treatment [11]. In the other patient, treatment and outcome were not reported [13]. Another patient developed disseminated disease after extensive wounds and died despite extensive debridement and antifungal treatment [14].

While mucormycosis in patients with diabetes mainly causes rhinocerebral and sino-orbital infections, patients with malignancy typically develop pulmonary infections. The extensive angioinvasive properties result in vessel thrombosis, tissue necrosis and haematogenous dissemination. Furthermore, ischaemic necrosis prevents leukocyte and drug penetration [4].

In our case, host factors and imaging (representing possible IFD $[8,15])$ led to the initiation of antifungal treatment. The growth of Aspergillus fumigatus from BAL fluid retrospectively most likely represents airway colonisation, reflecting the low specificity of cultivation of moulds from BAL fluid [1]. The detection of hyphae from otherwise sterile material and molecular analyses led to the diagnosis of proven mucormycosis [9].

Currently no indirect methods are available facilitating the diagnosis [16], since cell walls of Mucorales spp. are lacking (1-3)-beta-D-glucan and galactomannan, which therefore cannot be used to diagnose invasive mucormycosis $[1,4,17]$.

Initial empiric antifungal therapy comprising caspofungin, conventional doses of liposomal amphotericin B and voriconazole did not prevent deterioration. After proof of mucormycosis, therapy was switched to a combination of high-dose liposomal amphotericin B and posaconazole; despite lacking clear evidence, we decided for combination 
therapy due to the extent of the pulmonary lesions and the poor prognosis of invasive mucormycoses. Due to the widespread diffuse character of the infiltrates, we abandoned thoracotomy and surgical debridement. Mucorales spp. are considered to be resistant against echinocandins as well as against most azoles like voriconazole, fluconazole and itraconazole $[7,18]$ and require higher doses of amphotericin B usually administered in the liposomal formulation (AmBisome $\left.{ }^{\circledR}\right)[6$, 19]. Early treatment with high doses of amphotericin $B$ is important since a delay results in a twofold increase in mortality leading to an overall mortality of up to $100 \%$ for patients with disseminated disease [4]. The optimal dosage has still not been determined. Many clinicians use the maximum tolerable dosages, condoning nephrotoxicity, while efficacy results from a phase II clinical trial of high-dose therapy with $10 \mathrm{mg} / \mathrm{kg} /$ day i.v. are still pending (http://clinicaltrials. gov/show/NCT00467883) [4]. Furthermore, posaconazole monotherapy is not recommended due to several reports on breakthrough mucormycoses under posaconazole prophylaxis [20].

In addition to antifungal treatment, the elimination of risk factors such as immunosuppressive therapies is essential for successful management of mucormycosis [4, 21]. As in our patient, a common risk factor is the treatment of haematologic and other malignancies, which on the other hand is vital to be cured. Therefore, it was crucial to continue treatment of ALL as soon as possible, despite invasive pulmonary mucormycosis. After initiation of adequate therapy for mucormycosis, we also reinitiated antileukaemic treatment with an interval treatment aligned to the ALL maintenance therapy and switched to the reinduction according to protocol 7 weeks later. The duration of treatment for mucormycosis remains unclear. ECIL recommends duration on an individual basis, but should continue for at least 6-8 weeks [22]. We gradually reduced dosages and prolonged intervals of AmBisome $\AA$ infusions during ALL maintenance therapy to minimise side effects and to enable ambulatory parenteral therapy during several months of treatment. Intermittent AmBisome ${ }^{\circledR}$ administration is considered to be an effective alternative to the standard regimen in preventing and possibly treating invasive fungal diseases, since it appears to accumulate in tissue [23, 24]. We used this approach for a long time therapy of 1.5 years. However, after 1 year of antifungal treatment and 10 months of ALL maintenance therapy, again progression of the infiltrates was seen, which underlines the importance of radiologic monitoring and constant adequate treatment until immune reconstitution clearly has been achieved. We reintensified and successfully continued antifungal treatment with intermittent AmBisome ${ }^{\circledR}$ until numerical immune reconstitution had been indicated by flow cytometry 7 months after the end of ALL treatment. We monitored our patient via intermittent thoracic MRI in addition to CT scans to reduce radiation. To discriminate active infection from tissue scarring, we also used FDG-PET imaging, which has been described to have valuable sensitivity in detecting fungal lesions [25]. To gain immune reconstitution, one might be misled to extensively shorten maintenance therapy. However, we shortened ALL therapy by only 2 months, since it had been clearly shown that shortening therapy for ALL from 18 to 12 months leads to significantly worse outcome [26].

In conclusion, proof of mucormycetes from otherwise sterile material is essential for adequate diagnostics and therapy. Continued treatment of ALL is as important as concurrent adequate (long-term) antifungal treatment, alongside radiologic and immunologic monitoring which are essential in managing these both potentially fatal diseases.

Funding Information Open access funding provided by Medical University of Graz.

\section{Compliance with ethical standards}

Conflict of interest The authors declare that they have no conflict of interest.

The authors have full control of all primary data and allow the journal to review this data if requested.

Open Access This article is distributed under the terms of the Creative Commons Attribution 4.0 International License (http:// creativecommons.org/licenses/by/4.0/), which permits unrestricted use, distribution, and reproduction in any medium, provided you give appropriate credit to the original author(s) and the source, provide a link to the Creative Commons license, and indicate if changes were made.

\section{References}

1. Lamoth F, Calandra T (2017) Early diagnosis of invasive mould infections and disease. J Antimicrob Chemother 72(suppl_1):i19-i28

2. Dorin J, D'Aveni M, Debourgogne A, Cuenin M, Guillaso M, Rivier A, Gallet P, Lecoanet G, Machouart M (2017) Update on Actinomucor elegans, a mucormycete infrequently detected in human specimens: how combined microbiological tools contribute efficiently to a more accurate medical care. Int J Med Microbiol 307(8):435-442

3. Roden MM et al (2005) Epidemiology and outcome of zygomycosis: a review of 929 reported cases. Clin Infect Dis 41(5):634-653

4. Katragkou A, Walsh TJ, Roilides E (2014) Why is mucormycosis more difficult to cure than more common mycoses? Clin Microbiol Infect 20(Suppl 6):74-81

5. Skiada A, Pagano L, and Groll AEA 2010 Zygomycosis in Europe analysis of 230 cases accrued by the registry of the European Confederation of Medical Mycology (ECMM) working group on zygomycosis between 2005 and 2007. Clin Microbiol Infect

6. Walsh TJ, Goodman JL, Pappas P, Bekersky I, Buell DN, Roden M, Barrett J, Anaissie EJ (2001) Safety, tolerance, and pharmacokinetics of high-dose liposomal amphotericin B (AmBisome) in patients infected with Aspergillus species and other filamentous fungi: maximum tolerated dose study. Antimicrob Agents Chemother 45(12): 3487-3496 
7. Dannaoui E (2002) In vitro susceptibilities of zygomycetes to conventional and new antifungals. J Antimicrob Chemother 51(1):45-52

8. Hoenigl M, Strenger V, Buzina W, Valentin T, Koidl C, Wolfler A, Seeber K, Valentin A, Strohmeier AT, Zollner-Schwetz I, Raggam RB, Urban C, Lass-Florl C, Linkesch W, Krause R (2012) European Organization for the Research and Treatment of Cancer/ Mycoses Study Group (EORTC/MSG) host factors and invasive fungal infections in patients with haematological malignancies. $\mathrm{J}$ Antimicrob Chemother 67(8):2029-2033

9. De Pauw B et al (2008) Revised definitions of invasive fungal disease from the European Organization for Research and Treatment of Cancer/Invasive Fungal Infections Cooperative Group and the National Institute of Allergy and Infectious Diseases Mycoses Study Group (EORTC/MSG) Consensus Group. Clin Infect Dis 46(12):1813-1821

10. Gomes MZ, Lewis RE, Kontoyiannis DP (2011) Mucormycosis caused by unusual mucormycetes, non-Rhizopus, -Mucor, and Lichtheimia species. Clin Microbiol Rev 24(2):411-445

11. Mahmud A et al (2012) Actinomucor elegans as an emerging cause of mucormycosis. J Clin Microbiol 50(3):1092-1095

12. Davel G, Featherston P, Fernandez A, Abrantes R, Canteros C, Rodero L, Sztern C, Perrotta D (2001) Maxillary sinusitis caused by Actinomucor elegans. J Clin Microbiol 39(2):740-742

13. Khan ZU, Ahmad S, Mokaddas E, Chandy R, Cano J, Guarro J (2008) Actinomucor elegans var. kuwaitiensis isolated from the wound of a diabetic patient. Antonie Van Leeuwenhoek 94(3): 343-352

14. Tully CC, Romanelli AM, Sutton DA, Wickes BL, Hospenthal DR (2009) Fatal Actinomucor elegans var. kuwaitiensis infection following combat trauma. J Clin Microbiol 47(10):3394-3399

15. Groll AH, Castagnola E, Cesaro S, Dalle JH, Engelhard D, Hope W, Roilides E, Styczynski J, Warris A, Lehrnbecher T (2014) Fourth European Conference on Infections in Leukaemia (ECIL-4): guidelines for diagnosis, prevention, and treatment of invasive fungal diseases in paediatric patients with cancer or allogeneic haemopoietic stem-cell transplantation. Lancet Oncol 15(8):e327e340

16. Dornbusch HJ, Strenger V, Kerbl R, Lackner H, Schwinger W, Sovinz P, Urban C (2005) Procalcitonin-a marker of invasive fungal infection? Support Care Cancer 13(5):343-346

17. Egger $\mathrm{M}$ et al (2018) False positive serum levels of (1-3) - B-Dglucan after infusion of intravenous immunoglobulins and time to normalisation. J Inf Secur 76(2):206-210
18. Strenger V, Farowski F, Müller C, Hofer N, Dornbusch HJ, Sperl D, Lackner H, Benesch M, Urban C (2017) Low penetration of caspofungin into cerebrospinal fluid following intravenous administration of standard doses. Int J Antimicrob Agents 50(2):272-275

19. Strenger V, Meinitzer A, Donnerer J, Hofer N, Dornbusch HJ, Wanz U, Seidel MG, Sperl D, Lackner H, Schwinger W, Sovinz P, Benesch M, Urban C (2014) Amphotericin B transfer to CSF following intravenous administration of liposomal amphotericin B. J Antimicrob Chemother 69(9):2522-2526

20. Spellberg B, Ibrahim AS (2010) Recent advances in the treatment of mucormycosis. Curr Infect Dis Rep 12(6):423-429

21. Danion F, Aguilar C, Catherinot E, Alanio A, DeWolf S, Lortholary O, Lanternier F (2015) Mucormycosis: new developments into a persistently devastating infection. Semin Respir Crit Care Med 36(5):692-705

22. Skiada A, Lanternier F, Groll AH, Pagano L, Zimmerli S, Herbrecht R, Lortholary O, Petrikkos GL, on behalf of the third European Conference on Infections in Leukemia* (2013) Diagnosis and treatment of mucormycosis in patients with hematological malignancies: guidelines from the 3rd European Conference on Infections in Leukemia (ECIL 3). Haematologica 98(4):492-504

23. Ellis M (2008) New dosing strategies for liposomal amphotericin B in high-risk patients. Clin Microbiol Infect 14(Suppl 4):55-64

24. Bochennek K, Tramsen L (2011) Liposomal amphotericin B twice weekly as antifungal prophylaxis in paediatric haematological malignancy patients. Clin Microbiol Infect 17:1868-1874

25. Hot A, Maunoury C, Poiree S, Lanternier F, Viard JP, Loulergue P, Coignard H, Bougnoux ME, Suarez F, Rubio MT, Mahlaoui N, Dupont B, Lecuit M, Faraggi M, Lortholary O (2011) Diagnostic contribution of positron emission tomography with [18F]fluorodeoxyglucose for invasive fungal infections. Clin Microbiol Infect 17(3):409-417

26. Schrappe M, Reiter A, Zimmermann M, Harbott J, Ludwig WD, Henze G, Gadner H, Odenwald E, Riehm H (2000) Long-term results of four consecutive trials in childhood ALL performed by the ALL-BFM study group from 1981 to 1995 . Berlin-FrankfurtMunster. Leukemia 14(12):2205-2222

Publisher's note Springer Nature remains neutral with regard to jurisdictional claims in published maps and institutional affiliations. 